\title{
The Effectiveness of Mathematics in Physics of the Unknown
}

\author{
Alexei Grinbaum \\ CEA-Saclay/IRFU/LARSIM, 91191 Gif-sur-Yvette, France \\ Email alexei.grinbaum@cea.fr
}

\begin{abstract}
If physics is a science that unveils the fundamental laws of nature, then the appearance of mathematical formulae in its language can be surprising or even mysterious. This was Eugene Wigner's argument in 1960. I show that another approach to physical theory accommodates mathematics in a perfectly reasonable way. To explore unknown processes or phenomena, one builds a theory by employing fundamental principles as constraints within a general mathematical framework. Such studies of the unknown, or blackbox models, demonstrate the unsurprising effectiveness of mathematics on the example of Einstein's principle theories, the $S$-matrix approach in quantum field theory, effective field theories, and device-independent approaches in quantum information.
\end{abstract}

\section{Introduction}

"The enormous usefulness of mathematics in the natural sciences is something bordering on the mysterious... [T] here is no rational explanation for it," wrote Eugene Wigner in a well-known article in 1960 [51]. Above all, this "unreasonable" effectiveness manifests itself in physics. The latter, for Wigner, is devoted to "discovering the laws of inanimate nature." This view of physics, widespread but also challenged several times during the 20th century, relies on the concept of "law of nature" in a fundamental way. Any such law applies to one or several kinds of inanimate matter and describes their dynamical evolution. Physics is seen, then, as a study of natural phenomena by first deducing and subsequently applying the corresponding general laws. It may indeed seem mysterious that mathematical formulae should provide a faithful expression of such laws.

I submit that the effectiveness of mathematics in the natural sciences is perfectly reasonable and rational if one adopts a different view of physical theory. This view applies whenever the object of study involves phenomena or processes whose nature remains unknown. In these circumstances, physicists are not in position to say what kind of matter is involved but they are nevertheless eager to build a theory. For this, they employ fundamental principles, which are tasked with limiting the possibilities in theoretical description of unknown facts. For short, this approach will be named 'blackbox models.' The main property of the blackbox approach is that physical theory built in accordance with it is to be constrained by universal principles rather than dynamical laws. On this point it stands in opposition to Wigner's view but at the same time it remains sufficiently widespread, with applications spanning more than a century of research 
work that gave birth to new physical theories and discoveries. I illustrate the importance of this physics of the unknown on four examples: Einstein's principle theories (Section 2), S-matrix (Section 3), effective field theories (Section 4), and device-independent approaches (Section 5). On the basis of the case studies I will argue that the effectiveness of mathematics in blackbox models is neither surprising nor unreasonable. In fact, their success depends on mathematics as a driving force of theoretic construction.

\section{Principle theories}

In 1919 Einstein made a well-known distinction between principle and constructive theories [19]. Constructive theories match Wigner's view of physics: they contain dynamical laws describing the behaviour of particular kinds of matter, like Newton's laws do for the movement of rigid bodies. By contrast, a principle theory, e.g., Einstein's own special relativity, is a theory derived from simple postulates. It does not begin with an assumption about the type of matter or its dynamics; these become consequences of the postulates rather than theoretical prerequisites. The postulates are formulated as universal physical principles and are expressed in the formalism as mathematical axioms.

For example, the relativity principle or the upper limit on velocities given by the speed of light play the role of fundamental principles in Einstein's relativity theory. A modern avatar of these postulates, called no-signalling, stipulates that in an experimental setting with two observers the choice of measurement by one party must not influence the statistics of the outcomes registered by a different party. It is widely used in deviceindependent approaches for introducing constraints on operations with quantum information (see Section 5). The interest of this formulation is that it is entirely non-dynamical: no-signalling is an algebraic condition expressed in the language of conditional probability. At best it receives a kinematic - but not a dynamical - expression.

To use Einstein's own words about principle theories, the principles are employed in them in order to "narrow the possibilities" [17]. This means that one should begin the model-building exercise by adopting a very inclusive framework that can encompass the unknown phenomena in question but also much more. This framework may possibly extend beyond what has been or can be experimentally observed at the current stage of technological development. The point of starting with such a broad framework is to accommodate a yet unspecified theory with unpredictable empirical consequences. Principles, then, limit the possibilities offered by the broadness of the framework and serve to narrow it down to a particular model. For example, no-signalling excludes faster-thanlight travel in a geometric framework with a preselected spacetime, either Euclidean or Minkowski, or in the Riemannian way of introducing a spacetime manifold. In a non-dynamical framework which does not begin with a geometric object, the very notion of 'travel' might be undefined. Here, the no-signalling principle helps to make sure that a purely algebraic model will not produce a contradiction with the theory of relativity when it is 
applied for the description of real-world phenomena. The impossibility of faster-than-light signalling is "elevated" [23, p. 88] to the status of universal postulate even in the absence of geometric assumptions. It then becomes a fundamental principle of nature and a constitutive feature of physical theories.

Einstein's own road to the distinction between principle and constructive theories was a challenging one. After his 1905 article describing the photoelectric effect in terms of light quanta [18], his belief in the fundamental character and the exact validity of Maxwell's electrodynamics was destabilized. As he wrote in the 1949 Autobiographical Notes,

Reflections of this type [on the dual wave-particle nature of radiation] made it clear to me as long ago as shortly after 1900, i.e., shortly after Planck's trailblazing work, that neither mechanics nor electrodynamics could (except in limiting cases) claim exact validity. By and by I despaired of the possibility of discovering the true laws by means of constructive efforts based on known facts. [20, p. 51, 53]

This "desperation" led Einstein to special relativity. To find the theory, it was necessary to look for one that would not be based on "known facts." Special relativity, indeed, remains mute on the issue of material constitution of the rods and clocks that act as its measurement devices.

There is good evidence that Einstein believed that this lack of constructivity was a disadvantage and that principle theories did not offer a satisfactory understanding of physics $[11,24]$. This claim has been challenged recently via a comparison with James Jeans's position [32] but another, more seasoned critique focuses on the status of general relativity. According to Brown, it should be seen as a constructive theory since it contains a dynamical law [10]. Without entering the debate on relativity, I would like to emphasize the importance of the argument from explanatory power. The capacity to explain phenomena was uncontroversially ascribed by Einstein only to constructive theories: "When we say we have succeeded in understanding a group of natural processes, we invariably mean that a constructive theory has been found which covers the processes in question " [19]. Einstein wished to build an explanatory account based on known facts but despaired to do so. In his time and later, the desideratum to obtain a constructive theory as a replacement of principle-based special relativity never came to be realized.

To be sure, constructive theories are still widely in use. What has changed since the time of Einstein's tergiversations is that principles theories are now taken to be explanatory as well as capable of giving an understanding of physics on a par with constructive theories. The principle-based approach has been recognized as a legitimate way of doing physics. Its key method: the choice of a broad framework and its subsequent narrowing down through limiting principles, is at the same time an application of mathematics to physics and the enabling force behind theory-building and physical explanation. A conjunction of these two factors showcases the first examples of physical theory in which it is perfectly reasonable to assign a central methodological and explanatory place to mathematics. 


\section{S-matrix}

In the years before quantum electrodynamics and subsequently quantum chromodynamics were fully developed it had not been clear that a fieldtheoretic approach would be successful in accounting for the electromagnetic, the weak and the strong interactions. In the early 1950s, for example, it was not obvious to the physics community whether the method of quantum field theory (QFT) based on gauge symmetry would be an appropriate framework for building the theory of strong interactions. A similar uncertainty plagued quantum electrodynamics a decade earlier. In 1954, same year as the work by Yang and Mills, during a conference discussion in the presence of Oppenheimer, Gell-Mann, Fermi, Wick, and Dyson, Goldberger challenged the applicability of QFT methods to nuclear interactions. Surprisingly, nobody in the audience spoke to the contrary [35]. This episode was still remembered in the 1970s as a typical example of early doubts about the future of quantum field theory [2].

The doubts about the applicability of QFT were prevalent because of renormalizability issues. In response physicists began to look for methods to build a theory that did not assume any known particle content leading to divergencies. The main idea of this approach was borrowed from Heisenberg's philosophical program in the 1920s, which prescribed that a theory should focus only on observable quantities. This idea proved to be extremely successful in the discovery of quantum mechanics [28]. The hope was that the same approach would again produce a crucial insight. As Weinberg wrote, the physicists of the generation before his own believed that "by using principles of unitarity, analyticity, Lorentz invariance and other symmetries, it would be possible to calculate the $S$-matrix, and you would never have to think about a quantum field" [ 48 , p. 248]. Indeed, history has largely followed this prescription in developing the way in which our current physical theories with unknown particle content are constructed. One detail of this approach presents a particular philosophical interest. For a theoretician, the central question bears on the mathematical content of the theory: what mathematical objects should one use? Furthermore, what physical constraints can one impose on such objects? The success of the theory directly depends on finding an appropriate mathematical framework and tools.

In 1937 John Wheeler introduced one such mathematical object, which he called the scattering matrix, later to be known as $S$-matrix [50]. Wheeler's initial intent was to develop a mathematical method of "resonating group structure" that would allow one to build a description of the whole interacting system of elementary particles from the knowledge of its parts. This did not fully work out. Wheeler, however, obtained a result suggesting that the problem as he had formulated it could in fact be bypassed: "The connection which we have obtained between the scattering and disintegration cross sections does not depend for its validity on the accuracy of what we have called the method of resonating group structure." The scattering matrix that involved the cross sections depended only on some general asymptotic properties but not on the details of the interacting compound system. Among the general arguments used by Wheeler one mainly finds symmetry considerations credited by him to Bohr and Jor- 
dan. In the wake of Heisenberg's quantum mechanics, Wheeler's work provided a new example of a physical theory of unknown interactions, which involved exclusively the observables. It was built through the introduction of a new mathematical object. Wheeler published the results but did not pursue his method further; only much later did his scattering matrix become known as a precursor of the $S$-matrix theory of strong interactions [34, p. 990].

Between 1942 and 1944 Heisenberg, who did not know about Wheeler's work, wrote a series of three articles in Zeitschrift für Physik explicitly pursuing the goal of building a theory of unknown physics. The reason why the constructive physical content had to be taken as unknown, according to Heisenberg, was that the theory could change in the future:

In view of the later alteration [Abänderung] of the theory, the present investigation attempts to isolate from the conceptual scheme of the quantum theory of wave fields those concepts which probably will not be affected by the future changes [in the theory of elementary particles] and which may therefore represent and integral part [Bestandteil] also of the future theory. [30]

The concepts that Heisenberg thought not be affected by a future theory change were the observable quantities. He admitted although, in an indisputable influence of his earlier discussions with Einstein, that 'only the final theory will decide which quantities are "really observable." As early as 1938, simultaneously with Wheeler but independently, Heisenberg wrote:

Perhaps one may remember to advantage, in attempting to find new concepts, that in mathematical formulae, we are now confronted with the tast of finding computational rules, by which we can connect the cross sections... [29]

This stance, to quote the historian Helmut Rechenberg, was a consequence of the fact that "one did not yet know how to formulate a divergence-free theory describing elementary particles" [42]. Heisenberg's conviction was that the right theory would contain a minimal length. It was not immediately clear, however, how one was supposed to introduce such minimal length in QFT. Heisenberg reasonably believed that the asymptotic results, because they belong among observable quantities that the theory must be able to predict, should remain independent of the minimal length. While working out a complete theory remained a matter for future research, it was possible to introduce a direct connection between the momenta and the energies of free particles and the scattering and reaction cross sections. The connection was to be expressed mathematically: "It seemed appropriate to find a mathematical [our emphasis - AG] object capable of housing these observable quantities. Heisenberg realized that the momentum space kernel of the probability amplitude for transitions between free particle states was the object he wanted" [27]. Thus Heisenberg introduced a unitary 'characteristic' $S$-matrix becoming the founding father the the $S$-matrix approach in theoretical physics.

Heisenberg's $S$-matrix met fierce opposition from Wolfgang Pauli. He believed that it could not be fundamental, because the way the approach 
was constructed did not rely on arguments from simplicity and, in fact, produced a result that was anything but simple:

In general I have arrived at the opinion that the $S$-matrix is not a concept, of which we may expect that it occurs in a future theory as a primary fundamental concept. It indeed has the character of something complicated and derived and therefore might hardly be suitable to lead us beyond the present wave mechanics. [36]

This lack of simplicity underwrote Pauli's conviction that the $S$-matrix could not be a part of the laws of nature. It seems that Pauli believed for the general reasons of mathematical elegance that a law of nature should have a simple expression. He then concluded:

The $S$-matrix, although it might exist in a future theory, seems to be completely unfit to constitute the point of departure for a [new] theory. It is not the quantity which will occur in the general laws of nature, but a late consequence of them. [37]

This reveals the tension between two approaches to physical theory, each pushing toward a different role of the mathematical formalism. Pauli wished to have a theory containing laws of nature, i.e., dynamical rules of evolution of particular kinds of matter. If a law is found, e.g., describing light quanta, and if this law has a mathematical expression, then it is perfectly legitimate to wonder why mathematics would be so effective in describing the behaviour of light quanta. It is even more surprising that mathematics is equally effective in describing the evolution of directly perceivable objects like tables or chairs. Whatever answer one may give to this Wignerian wonder, the theory in question is a constructive theory in Einstein's terms.

The situation is different with principle theories. They explore unknown territories, which cannot yet be accounted for in terms of a particular kind of matter, let alone a law of its dynamical evolution. The $S$-matrix, as Wheeler discovered, bypasses the problem of "resonating group structure," which would describe the content of the theory in terms of interacting particles. Similarly, Heisenberg's focus on observable quantities does not require a physical description of how one such observable gets dynamically converted into another. The middle ground can remain unknown - a black box - while mathematical relations will still be available describing the relation between the observables. This is a clear sign that mathematics in a principle theory is not playing the role of underwriting the laws of nature, as Wigner thought, but rather of letting a theory of the unknown to be built in the first place. When Gregor Wentzel called the $S$-matrix program "very incomplete-it is like an empty frame for a picture yet to be painted" [49], he believed to be giving a pejorative assessment of Heisenberg's program. In fact, he put his finger on the main feature of principle theories: a physical theory is possible without filling in "an empty frame" or opening up a black box.

The $S$-matrix theory of nuclear interactions has become history after the advent of quantum chromodynamics but the $S$-matrix approach is still well and alive. In quantum gravity, for example, it is used for constructing 
low-energy models of supergravity from high-energy theories like string theory. String theory itself was discovered by Veneziano as a consequence of his work on the $S$-matrix approach, when he used general principles to correctly guess the unknown amplitudes satisfying duality properties, which described the excitations of a one-dimensional object [44]. One can use this all-encompassing theory of quantum gravity to construct lowenergy gravitational models with unknown physical content. This study of unknown territory requires the same tool as the one used by Heisenberg for exploring the unknown land of QFT, the $S$-matrix:

Such an S-matrix, which is tightly constrained by properties such as unitarity and analyticity, can be a very powerful way to summarize our ignorance of a theory. ... We might anticipate that such study in the context of gravity, supplemented by additional physical input, could bear important fruit. [25]

Thus the anticipated physics is always mathematical.

\section{Effective field theories}

The $S$-matrix approach only asked 'practical' questions about the yet unknown theory of strong interactions, formulated in the language of physical observables, and methodically avoided the need to have a full theory. In the effective field theory (EFT) approach the unknown is not the theory of nuclear interactions but new physics beyond the Standard Model. With little prospect for distinguishing in the near future between the different alternatives for this new physics, EFT offers a method for developing a theory-independent approach, where all that matters about the new unknown physics are its observable effects. Just as $S$-matrix allows one to focus on observable quantities and disregard the quantum field, EFT relieves one from the need to worry about physical content of the highenergy theory. Indeed, EFT prescribes that the Lagrangian include all terms in the most general form compatible with symmetry principles. No particular physical content or physical meaning is assumed, while symmetry principles are employed as constraints.

The notion of renormalizability in the context of quantum field theory and its early representatives like quantum electrodynamics (QED) was developed in the mid-20th century by Bethe, Schwinger, Tomonaga, Feynman, and Dyson. The latter introduced crucial power-counting techniques for analyzing operator relevance. Since his 1949 work [15, 16] and up to 1970s renormalizability had been thought of as a necessary condition for a quantum field theory to make sense. Wilson's work on the renormalization group [52] has paved the way to a change of attitude toward renormalizability. This was mainly due to a change of attitude toward the reality of the renormalization cut-off. In the older understanding, the cutoff scale was a residue of abstract mathematics introduced with the only goal of avoiding infinities in summation series. The new appreciation of non-renormalizable theories came with the understanding that the cut-off could be taken as physical and corresponding to the limit of applicability of a given theory. New physics was to be expected beyond the cut-off scale 
$\Lambda_{N P}$. Thus the domain of applicability of particular QFTs has become clearly limited by a number denoting an energy scale. Quantum field theories began to be seen as effective field theories valid up to some frontier, rather than fundamental theories of nature. Wilson's work and Weinberg's reintroduction of EFTs as useful theories with 'phenomenological Lagrangians' [45, 47, 46] boosted this new view on EFTs.

Much of the historic development of EFTs focused on the top-down approach, which stipulates that the fundamental physical theory is known but is inapplicable for practical purposes. This inapplicability may be due to the complexity of high-energy theory or, as in the case of EFT in condensed matter physics, to heuristic arguments: "Even when one knows the theory at a microscopic level (i.e., the fundamental theory), there is often a good reason to deliberately move away to an effective theory" [43]. A typical example from particle physics is the chiral perturbation theory, which gives a low-energy approximation of quantum chromodynamics (QCD) in the light quark sector (for a review see [38]). Even when physical content is known, then, it is often instructive and necessary to build a physical theory as if this content remained unknown. This underlines the importance of blackbox models: the history of the top-down approach, treating the known as if it were unknown, goes back to the Euler-Heisenberg calculation in the 1930s of photon-photon scattering at small energies within the framework of Dirac's quantum field theory [22, 21, 31].

High-energy physics today uses an alternative EFT approach, sometimes called 'bottom-up'. Its popularity reflects a change in the way in which EFTs are now conceived. Today physicists tend to think of all physical theories, including the Standard Model, as EFTs with respect to new physics at higher energies. Blackbox models have become universal: any quantum field theory is, to some extent, a theory of the unknown.

A typical model-building scenario, following Wilson, starts with a Lagrangian of an effective field theory (EFT) valid up to scale $\Lambda$. This Lagrangian can be generally written as a sum over local operator products:

$$
\mathcal{L}=\sum_{n=0}^{\infty} \frac{\lambda_{n}}{\Lambda^{n}} \mathcal{O}_{n} .
$$

Coefficients $\lambda_{n}$ are coupling constants. They encode information on the physics at scales higher than $\Lambda$ and can be fixed experimentally or through a calculation by the renormalization group if the underlying high-energy theory is known. The only constraints on the form of operator product terms $\mathcal{O}_{n}$ come from the symmetries of the theory. The tree level of the power series in $\frac{1}{\Lambda}$ is obtained by the usual Standard Model calculation. Effects of new physics appear in loop corrections and influence the value of coupling constants $\lambda_{n}$. The main value of Lagrangian (1) for highenergy physics is that it can be used to study low-energy effects of new physics beyond the Standard Model without having to specify what this new physics actually is.

For example, consider a 'top-down' electroweak EFT that reproduces the Standard Model for the light degrees of freedom (light quarks, leptons and gauge bosons) as long as energies involved are small compared with the Higgs mass [38]. This EFT is Higgless in the sense that it cuts off the 
Higgs sector by a choice of $\Lambda$. The lowest order effective Lagrangian fixes the masses of $Z$ and $W$ bosons at tree level and does not carry information on the underlying symmetry breaking $S U(2)_{L} \times U(1)_{Y} \rightarrow U(1)_{\text {QED }}$. At the next order the most general effective chiral Lagrangian with only gauge bosons and Goldstone fields,

$$
\mathcal{L}_{\mathrm{EW}}^{(4)}=\sum_{i=0}^{14} a_{i} \mathcal{O}_{i},
$$

contains 15 independent operators. This complexity is essential as it stems from the requirement that we use the most general form of the Lagrangian compatible with symmetry principles. Gell-Mann has even formulated this rule as a "totalitarian principle" which states that everything which is not forbidden is compulsory [9]. For Lagrangian (2), constraints from symmetry include invariance with respect to $C P$ and $S U(2)_{L} \times U(1)_{Y}$. Also, three of the fifteen operators vanish as a consequence of the equations of motion under the assumption of light fermions. With the remaining terms, one finds various effects such as the usual electroweak oblique corrections (6 operators involved at the bilinear, 4 at the trilinear and 5 at the quartic levels), corrections to rare $B$ and $K$ decays, the $C P$-violating parameter, etc. Thus, the approximation of a very large Higgs mass in the Standard Model gives an EFT which possesses phenomenological predictive power, providing an easier way to perform calculations than the complete Standard Model Lagrangian.

As if he were developing an argument to counter Pauli's critique of Heisenberg's $S$-matrix, Weinberg insists that the absence of any assumption of simplicity about the EFT Lagrangian is what makes the EFT method so efficient [48, p. 246]. He further supports the parallel by claiming that "the $S$-matrix philosophy is not far from the modern philosophy of effective field theories". However, he also adds a critique of $S$-matrix: "More important than any philosophical hang-ups was the fact that quantum field theory didn't seem to be going anywhere in accounting for the strong and weak interactions". The $S$-matrix was the only rational reaction to a situation in which no one knew what language to use, nor in which direction to look for a theory of the strong and weak interactions. This was a despair quite analogous to Einstein's unease when he had realized that the theory he was developing could not be based on known facts (Section 2). Similarly, today we do not know whether supersymmetry, or extra dimensions, or yet another model, will turn out to be the right solution for new physics. However, the blackbox approach to unknown phenomena has been generalized in EFT to the point where we apply it above and beyond any situation of despair. It has become a usual, and arguably a normative, tool in quantum field theory. Like Einstein or Heisenberg, we resort to a language that does not require knowledge of the dynamical laws or constitutive types of matter. Unlike Einstein or Heisenberg, we treat this situation as perfectly reasonable. The method of building an EFT that starts from a general mathematical framework of gauge theory, then proceeds with a Lagrangian compatible with the constraints coming from symmetry principles, is neither a surprising nor a scandalous jump as Pauli may have thought about Heinsenberg's $S$-matrix. That mathe- 
matics plays an effective role in physics of the unknown, seen from the point of view of effective field theories, is a new normal.

\section{$5 \quad$ Device-independent models}

Quantum cryptography works with systems of "unspecified character" [4] or "unknown nature" [5]. This is performed in a device-independent approach: a theoretical investigation that does not rely on the knowledge of laws governing the systems' behaviour. A conventional 'device' refers here to any process or apparatus described by an operational theory, whether classical or quantum, which is explicitly designated. This terminology was first introduced by Mayers and Yao [33], who developed device-independent quantum cryptography with imperfect sources. Over the years quantum cryptography has developed an array of such methods for dealing with adversaries which, via action upon sources, effectively turn systems into untrusted entities. Device-independent protocols are important for randomness generation [14, 39], quantum key distribution [6], estimation of the states of unknown systems [5], certification of multipartite entanglement [4], and distrustful cryptography [1]. Some of these cryptographic protocols have found a broader use in quantum information, e.g., device-independent tests performed on Bell inequalities or on the assumption that superluminal signaling is impossible [3].

In full generality, device-independent models are defined as a set of $n$ parties, each of which 'selects' a measurement setting or 'places' an input value $x_{1} \in \mathcal{X}_{1}, \ldots, x_{n} \in \mathcal{X}_{n}$ respectively, and 'subsequently' 'obtains' an output value or a measurement result $a_{1} \in \mathcal{A}_{1}, \ldots, a_{n} \in \mathcal{A}_{n}$. The sets $\mathcal{X}_{1}, \ldots, \mathcal{X}_{n}$ and $\mathcal{A}_{1}, \ldots, \mathcal{A}_{n}$ are alphabets of finite cardinality. The verbs used in these expressions merely convey an operational meaning of the inputs and outputs; they do not imply that any party exercises free will or has conscious decision-making procedures. The term 'subsequently' introduces a local time arrow pointing from each party's input to its output. Although such local time arrows seem quite intuitive, in full generality they need not be assumed either. A fully general setting requires, therefore, that absolutely nothing be postulated about the way inputs are transformed into outputs, except two conditions: a) these two types of data are clearly distinguished; $b$ ) the process of transformation is physical. Physics is contained in the probability distribution $\mathbf{p}=P\left(a_{1}, \ldots, a_{n} \mid x_{1}, \ldots, x_{n}\right)$ (Figure 1).

All device-independent models studied in the literature introduce further constraints on $\mathbf{p}$. The most frequent one is the no-signalling principle discussed in Section 2: a choice of measurement by one party must not influence the statistics of the outcomes registered by a different party. Mathematically, the distribution $\mathbf{p}$ is non-signalling if and only if all oneparty marginal probabilities are functions of their respective inputs $x_{i}$ :

$$
P\left(a_{i} \mid x_{1}, \ldots, x_{n}\right)=P\left(a_{i} \mid x_{i}\right) .
$$

Although very common, this assumption is not universal: when deviceindependent methods are used to test general causal inequalities, the impossibility of signalling is not a prerequisite [7]. 


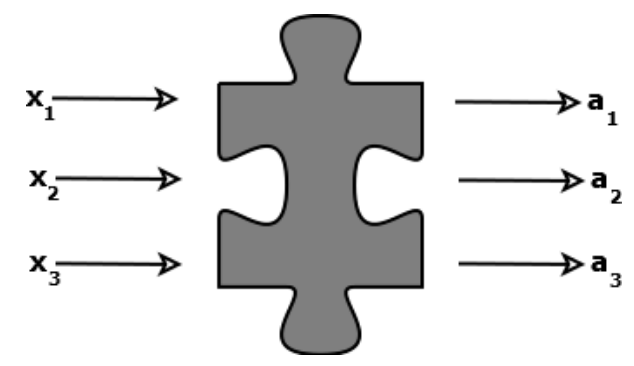

Figure 1: In the case of $n=3$ parties, physics is fully contained in the probabilities $\mathbf{p}=P\left(a_{1} a_{2} a_{3} \mid x_{1} x_{2} x_{3}\right)$.

One of the earliest examples of device-independent methods in quantum information involves what is literally called a box. The no-signalling constraint was studied by Popescu and Rohrlich [41] through the introduction of a non-local, or Popescu-Rohrlich (PR), box describing unknown processes which connect the inputs $x, y \in\{0,1\}$ and the outputs $a, b \in\{0,1\}$ of two parties according to the joint distribution:

$$
P(a b \mid x y)= \begin{cases}1 / 2: & a+b=x y \bmod 2 \\ 0: & \text { otherwise. }\end{cases}
$$

While a PR-box is a general algebraic framework designed to go beyond quantum theory, the application of the no-signalling principle implies that this box nevertheless respects the laws of special relativity. Its deviceindependent non-local structure accommodates a violation of the Tsirelson bound [12] by reaching the maximum amount of correlations in the $\mathrm{CHSH}$ inequality $[8,13]$.

Hailed as a "very important recent development" [40], device-independent models are characterized by the absence of assumptions about the internal workings of the box. Its 'interior' is not described by a particular physical theory. The box is unknown territory which, since it is assumed to be of interest for physical theory, is also a territory of science. The entire setup belongs within the boundaries of physics; at the same time, it opens a possibility to redefine these very boundaries [26]. This redefinition is achieved via the use of mathematics: particular mathematical constraints are applied in the general operational probabilistic framework, whose only elementary notions are the inputs and the outputs. Not only is the effectiveness of mathematics unsurprising; it becomes a driving force propelling the development of physical theory.

\section{Conclusion}

Wigner's point about the unreasonable effectiveness of mathematics in physical theory was that nothing, in principle, urges nature to be mathematical. Wigner did not seek to ground his statement in a particular philosophical system but rather expressed the immediate surprise of anyone who discovers that mathematical formulae can correctly describe the 
objective world or reality outside human mind. Evidently, a Pythagorean or a neo-Platonist would not be so surprised, for these philosophical systems put the number among the fundamental constitutive principles of nature. But Wigner's amazement remain valid nevertheless.

In theoretical terms the Wignerian wonder can be understood as a question about what we observe or manipulate in the outside world: why are these objective phenomena and inert matter described by mathematical laws? But physical theory does not always deal with phenomena or matter that are already known or available. Often it must explore the unknown. In this case, one of the tasks of physical theory is to determine the kind of matter involved or the type of phenomena that can be observed in support of new theory. The impulse for creating such a theory comes from the urge to study a new and unknown territory. The origin of the urge usually lies in the dissatisfaction with old theories rather than any precise ideas about where to look for a new one. On other occasions old and complete theories are too complex or unsuited for the needs of solving particular problems. It is then reasonable to treat what is known as if it were unknown. Physics of the unknown establishes itself as an exploratory instrument also within the territory of known facts and phenomena.

Physical theory of the unknown is a blackbox model trying to find a link between the inputs and the outputs of the box. This is the language of the device-independent approach. Equivalently, one may say with Heisenberg that it only operates with observable quantities. Wheeler expressed the same idea by focusing his introduction of the $S$-matrix on asymptotically free particle states. In effective field theories, the unknown high-energy theory is replaced by operators describing all possible effects observable at a given energy scale.

What is unknown is placed in a black box, which the theory does not necessarily seek to open up. As Einstein's principle theory show, this approach does not and often cannot help to uncover the content of the box. In spite of its non-constructive character, it can still be predictive and explanatory. Explanation in this case comes, not from the knowledge of what is inside the box, but from the postulates that constrain the connection between the inputs and the outputs. If a theory is successful and makes correct predictions, then these principles become our best candidates for fundamental principles of nature. This new knowledge about the world does not come in the form of a new dynamical law for a new kind of matter. Instead we learn a new principle, whose status is established through an enquiry grounded in mathematics.

The connection between the inputs and the outputs of a black box in a device-independent approach is inherently mathematical. To develop a principle theory means to identify the right mathematical link, hence a right mathematical concept and often a right mathematical language for connecting the inputs and the outputs. This search is performed in the space of theories rather than in an empirical world of physical experimentation. A success, for sure, can only be proclaimed if the identified mathematical object helps to make empirical predictions or explain phenomena that had previously stayed unaccounted. Whenever one achieves such success, the mathematical nature of this connection between the inputs and the outputs also provides constitutive power for the theory. 
Contrary to Wigner's attitude of mystery and amazement, the effectiveness of mathematics in describing the physics of the unknown-new and uncharted territory of science or nature yet unexplored - cannot but be deemed reasonable and unsurprising.

\section{References}

[1] N. Aharon, S. Massar, S. Pironio, and J. Silman. Device-independent bit commitment based on the CHSH inequality. New J. of Physics, 18:025014, 2016, arXiv:1511.06283.

[2] T. Appelquist and J. D. Bjorken. Weak interactions at high energies. Phys. Rev. D, 4(12):3726-3737, 1971.

[3] J.-D. Bancal. On the Device-Independent Approach to Quantum Physics: Advances in Quantum Nonlocality and Multipartite Entanglement Detection. Springer, Geneva, 2013.

[4] J.-D. Bancal, N. Gisin, Y.-C. Liang, and S. Pironio. Device-independent witnesses of genuine multipartite entanglement. Phys. Rev. Lett., 106:250404, 2011.

[5] C.-E. Bardyn, T. C. H. Liew, S. Massar, M. McKague, and V. Scarani. Device-independent state estimation based on Bell's inequalities. Phys. Rev. A, 80:062327, 2009.

[6] J. Barrett, L. Hardy, and A. Kent. No signaling and quantum key distribution. Physical Review Letters, 95(1):010503, June 2005.

[7] Ä. Baumeler and S. Wolf. Perfect signaling among three parties violating predefined causal order. In Proceedings of 2014 IEEE International Symposium on Information Theory (ISIT), pages 526-530, 2014.

[8] J. Bell. On the Einstein-Podolsky-Rosen paradox. Physica, 1:195-200, 1964.

[9] O. M. P. Bilaniuk and E. C. G. Sudarshan. Particles beyond the light barrier. Physics Today, 22:43-51, May 1969. This is the first known reference in press. Attribution to Gell-Mann is however indisputable.

[10] H. Brown. Physical Relativity: Space-Time Structure from a Dynamical Perspective. Oxford University Press, Oxford, 2005.

[11] H. Brown and C. Timpson. Why special relativity should not be a template for a fundamental reformulation of quantum mechanics. In W. Demopoulous and I. Pitowsky, editors, Physical Theory and Its Interpretation, pages 29-42. Springer, Amsterdam, 2006, arXiv:quant-ph/0601182.

[12] B. S. Cirel'son. Quantum generalizations of Bell's inequality. Lett. Math. Phys., 4(2):93-100, 1980.

[13] J. Clauser, R. Holt, M. Horne, and A. Shimony. Proposed experiment to test local hidden-variable theories. Phys. Rev. Lett., 23:880-884, 1969.

[14] R. Colbeck. Quantum And Relativistic Protocols For Secure Multi-Party Computation. PhD thesis, University of Cambridge, December 2006.

[15] F. Dyson. The radiation theories of Tomonaga, Schwinger, and Feynman. Phys. Rev., 75:486-502, 1949. 
[16] F. Dyson. The S-matrix in quantum electrodynamics. Phys. Rev., 75:1736$1755,1949$.

[17] A. Einstein. Address to a scientific meeting in Zurich, 1911. Cited in: P. Galison, Einstein's Clocks, Poincaré's Maps. Empires of Time, Hodder and Stoughton, London, 2004, p. 268.

[18] A. Einstein. Über einen die Erzeugung und Verwandlung des Lichtes betreffenden heuristischen Gesichtspunkt. Ann. d. Phys., 17:132-148, 1905.

[19] A. Einstein. What is the theory of relativity? London Times, 1919. Reprinted in: A. Einstein, Ideas and Opinions, Crown Publishers, New York, 1982.

[20] A. Einstein. Autobiographical notes. In P. Schlipp, editor, Albert Einstein: Philosopher-Scientist, volume 7 of The Library of Living Philosophers, pages 1-94. Open Court, Illinois, 1949.

[21] H. Euler. Über die Streuung von Licht an Licht nach der Diracschen Theorie. Ann. der Physik, 418:398-448, 1936.

[22] H. Euler and B. Kockel. Über die Streuung von Licht an Licht nach der Diracschen Theorie. Naturwissenschaften, 23:246, 1935.

[23] M. Friedman. Dynamics of Reason. CSLI Publications, Stanford, 2001.

[24] M. Frisch. Mechanisms, principles, and Lorentz's cautious realism. Studies in the History and Philosophy of Modern Physics, 36:659-679, 2005.

[25] S. B. Giddings. The gravitational $S$-matrix. In 48th Course of the Erice International School of Subnuclear Physics, volume 48 of Subnuclear Ser., pages 93-147, 2013, arXiv:1105.2036.

[26] A. Grinbaum. How device-independent approaches change the meaning of physical theory. Studies in the History and Philosophy of Modern Physics, 2017, arXiv:1512.01035. In print.

[27] I. Grythe. Some remarks on the early S-matrix. Centaurus, 26:198-203, 1982.

[28] W. Heisenberg. Über quantentheoretische Umdeutung kinematischer und mechanischer Beziehungen. Zeit. f. Phys., 33:879-893, 1925.

[29] W. Heisenberg. Über die in der Theorie der Elementarteilchen auftretende universelle Länge. Ann. Phys., 424:20-33, 1938.

[30] W. Heisenberg. Die "beobachbaren Grössen" in der Theorie der Elementarteilchen. Zeit. f. Phys., 120:513-539, 1942. English translation in [34, p. 1030-1031].

[31] W. Heisenberg and H. Euler. Folgerungen aus der Diracschen Theorie des Positrons. Z. Phys., 98:714, 1936.

[32] M. Lange. Did Einstein really believe that principle theories are explanatorily powerless? Perspectives on Science, 22(4):449-463, 2014.

[33] D. Mayers and A. Yao. Quantum cryptography with imperfect apparatus. In FOCS 1998: Proceedings of the 39th Annual Symposium on Foundations of Computer Science, pages 503-509. IEEE Computer Society, Los Alamitos, CA, USA, 1998. 
[34] J. Mehra and H. Rechenberg. The Conceptual Completion and the Extensions of Quantum Mechanics 1932-1941. Epilogue: Aspects of the Further Development of Quantum Theory 1942-1999, volume 6 of The Historical Development of Quantum Theory. Springer, 2001.

[35] H. P. Noyes, editor. Proceedings of the Fourth Annual Rochester Conference on High Energy Nuclear Physics. The University of Rochester and The National Science Foundation, 1954.

[36] W. Pauli. Letter to W. Heisenberg, 9 september 1946. Cited in [42, p.566], 1946.

[37] W. Pauli. Letter to W. Heisenberg, 20 october 1948. Cited in [42, p.568], 1948.

[38] A. Pich. Effective field theory. In R. Gupta et al., editors, Proceedings of Les Houches Summer School of Theoretical Physics 'Probing the Standard Model of Particle Interactions', volume II, page 949, Amsterdam, 1999. Elsevier, arXiv:hep-ph/9806303.

[39] S. Pironio et al. Random numbers certified by Bell's theorem. Nature, 464:1021-1024, 2010.

[40] S. Popescu. Nonlocality beyond quantum mechanics. Nature Physics, 10:264-270, 2014.

[41] S. Popescu and D. Rohrlich. Nonlocality as an axiom for quantum theory. Foundations of Physics, 24:379, 1994, quant-ph/9508009.

[42] H. Rechenberg. The early $S$-matrix theory and its propagation (1942-1952). In L. M. Brown, M. Dresden, L. Hoddeson, and M. West, editors, Pions to quarks: Particle physics in the 1950s, pages 551-578. Cambridge University Press, Cambridge, 1989.

[43] R. Shankar. Effective field theory in condensed matter physics. pages 47-55. Cambridge University Press, 1999.

[44] G. Veneziano. Construction of a crossing-symmetric, Regge behaved amplitude for linearly rising trajectories. Nuovo Cim., A57:190-197, 1968.

[45] S. Weinberg. Dynamical approach to current algebra. Phys. Rev. Lett., 18:188-191, 1967.

[46] S. Weinberg. Phenomenological lagrangians. Physica, 96A:327-340, 1979.

[47] S. Weinberg. Precision tests of quantum mechanics. Phys. Rev. Lett., 62:485-488, 1989.

[48] S. Weinberg. Sokal's hoax. The New York Review of Books, XLIII(13):11$15,1996$.

[49] G. Wentzel. Recent research in meson theory. Rev. Mod. Phys., 19:1-18, 1947.

[50] J. Wheeler. On the mathematical description of light nuclei by the method of resonating group structure. Phys. Rev., page 1107, 1937.

[51] E. Wigner. The unreasonable effectiveness of mathematics in the natural sciences. Communications in Pure and Applied Mathematics, 13:1-14, 1960.

[52] K. G. Wilson. The renormalization group and strong interactions. Phys. Rev. D, 3:1818, 1971. 